\title{
Exact acceleration of complex real-time model checking based on overlapping cycle
}

\author{
Guoqing Wang ${ }^{1}$, Lei Zhuang ${ }^{\text {Corresp., } 1}$, Yu Song ${ }^{1}$, Mengyang He ${ }^{1}$, Ding Ma ${ }^{2}$, Ling Ma ${ }^{1,3}$ \\ ${ }^{1}$ School of Information Engineering, Zhengzhou University, Zhengzhou, Henan, China \\ 2 College of Information Science and Engineering, Henan University of Technology, Zhengzhou, Henan, China \\ 3 Digital Medical Image Technique Research Center, Zhengzhou University, Zhengzhou, Henan, China \\ Corresponding Author: Lei Zhuang \\ Email address: ielzhuang@zzu.edu.cn
}

When real-time systems are modeled as timed automata, different time scales may lead to substantial fragmentation of the symbolic state space. Exact acceleration solves the fragmentation problem without changing system reachability. The relatively mature technology of exact acceleration has been used with an appended cycle or a parking cycle, which can be applied to the calculation of a single acceleratable cycle model. Using these two technologies to develop a complex real-time model requires additional states and consumes a large amount of time cost, thereby influencing acceleration efficiency. In this paper, a complex real-time exact acceleration method based on an overlapping cycle is proposed, which is an application scenario extension of the parking-cycle technique. By comprehensively analyzing the accelerating impacts of multiple acceleratable cycles, it is only necessary to add a single overlapping period with a fixed length without relying on the windows of acceleratable cycles. Experimental results show that the proposed timed automaton model is simple and effectively decreases the time costs of exact acceleration. For the complex real-time system model, the method based on an overlapping cycle can accelerate the large scale and concurrent states which cannot be solved by the original exact acceleration theory. 


\section{Exact acceleration of complex real-time model 2 checking based on overlapping cycle}

3

\section{Guoqing Wang ${ }^{1}$, Lei Zhuang ${ }^{1}$, Yu Song ${ }^{1}$, Mengyang $\mathrm{He}^{1}$, Ding $\mathrm{Ma}^{2}$, Ling $\mathrm{Ma}^{1,3}$}

${ }^{1}$ School of Information Engineering, Zhengzhou University, Zhengzhou, Henan, China

${ }^{2}$ College of Information Science and Engineering, Henan University of Technology, Zhengzhou, Henan, China

${ }^{3}$ Digital Medical Image Technique Research Center, Zhengzhou University, Zhengzhou, Henan, China

Corresponding Author:

Lei Zhuang ${ }^{1}$

Science Avenue No. 100, Zhengzhou, Henan, 450001, China

Email address: ielzhuang@zzu.edu.cn

\section{Abstract}

When real-time systems are modeled as timed automata, different time scales may lead to substantial fragmentation of the symbolic state space. Exact acceleration solves the fragmentation problem without changing system reachability. The relatively mature technology of exact acceleration has been used with an appended cycle or a parking cycle, which can be applied to the calculation of a single acceleratable cycle model. Using these two technologies to develop a complex real-time model requires additional states and consumes a large amount of time cost, thereby influencing acceleration efficiency. In this paper, a complex real-time exact acceleration method based on an overlapping cycle is proposed, which is an application scenario extension of the parking-cycle technique. By comprehensively analyzing the accelerating impacts of multiple acceleratable cycles, it is only necessary to add a single overlapping period with a fixed length without relying on the windows of acceleratable cycles. Experimental results show that the proposed timed automaton model is simple and effectively decreases the time costs of exact acceleration. For the complex real-time system model, the method based on an overlapping cycle can accelerate the large scale and concurrent states which cannot be solved by the original exact acceleration theory.

\section{Introduction}

In real-time embedded systems, especially complex real-time control systems, discrete logic control and continuous time behavior depend on and influence each other. Take the Internet of things (IoT) gateway security system (Wang et al., 2018) as an example: Its control center generally has many different control modes to deal with diverse security risks, such as tampering, intrusion, and identity forging. Important system parameters (e.g., sensor status, monitoring 
40

41

42

43

44

45

46

47

48

49

50

51

52

53

54

55

56

57

58

59

60

61

62

63

64

65

66

67

68

69

70

71

72

73

74

75

76

77

78

instructions, and terminal feedback information) change continuously over time. To meet specific time constraints or parameter values in the IoT gateway security system, the management mode must be adjusted over time. The change rules of important parameters also differ by mode, and the response time to various events should be modified accordingly. In this type of system (Lee et al., 2019), logic control describes the logical control transformation of the system through models with high abstraction levels, such as finite state machine and Petri net. Time behavior can be simulated by clock variables and clock zone transformation. Between the two layers, signals of the continuous layer and control modes of the discrete layer are correlated and transformed by certain interfaces and rules.

Typically, test and simulation technologies are the main means of guaranteeing software quality; however, they cover problems when using the operating system as the main measure, which cannot guarantee test completeness. These approaches are thus incapable of traversing all states in a realtime system, leading to covert problems in system operations (Wang, Pastore \& Briand, 2019). In the field of security-related systems with zero tolerance for system error, using formal theory and technology for security authentication results in clear descriptions and avoids the complexity of safety verification. Formal description analysis and refinement have thus become a focus of recent research in related fields.

In real-time model checking, timed automata can model the temporal behavior of real-time systems (Pinisetty et al., 2017). Clocks describe the state transitions, and clock constraints serve as the theoretical basis for real-time system model checking (Han, Yang \& Xing, 2015). This approach can easily realize automatic combination and transformation with other methods. The method is widely used in polling control systems, railway interlocking systems, and similar applications. Due to clock variables, control programs and external environments often use different time measures, which can cause the number of states to increase exponentially when a timed automaton is transformed into a zone automaton. The reachability analysis algorithm generates many state fragments (Iversen et al., 2000; Chen \& Cui, 2016), resulting in a sharp increase in the state space and considerably prolonged detection time.

The acceleration technique is a reduction method used to solve the fragmentation problem following from time measurement differences. Dubout \& Fleuret (2013) applied an acceleration technique to linear target detection and effectively improved the detection performance. Jeong et al. (2014) applied an implicit Markov model as an improved framework to accelerate the inference model. For distributed and parallel computing, a workstation and a multicore processor were used to accelerate state-space searching (Konur, Fisher \& Schewe, 2013). Lin, Chen \& Xu (2017) studied an acceleration model using a Bayesian classifier by analyzing the behavior of heterogeneous population trends; results indicated that acceleration in the reliability assessment improved the analytic accuracy. The model checking of linear temporal logic (LTL) model was studied by Barnat et al. (2010), which employed computed unified device architecture for acceleration. Two SAT problem solvers were used to validate online models and accelerate the processing of complex behaviors (Qanadilo, Samara \& Zhao, 2013). 
79

80

81

82

83

84

85

86

87

88

89

90

91

92

93

94

95

96

97

98

99

100

101

102

103

104

105

106

107

108

109

110

111

112

113

114

115

116

117

118

The reachability problem is the first to consider in timed automata, which determines whether a path exists from its initial state to a target state. This problem can be solved by computing the zones that apply the abstraction technique in practice. State-of-the-art abstraction methods (Behrmann et al.,2006; Herbreteau, Srivathsan \& Walukiewicz, 2016) produce an approximation closer to the actual reachable clock valuation, which includes coarser abstractions. Exact acceleration is an excellent means of abstraction to reduce required storage space and can alleviate state-space explosion. For practical issues such as protocol validation (Zhang et al., 2013), IoT system modeling (Li et al., 2013), and smart contract security verification in blockchain (Cruz, Kaji \& Yanai, 2018; Grishchenko, Maffei \& Schneidewind, 2018), exact acceleration technology is an efficient way of minimizing required storage space and time.

When Iversen et al. (2000) used UPPAAL to verify the LEGO robotic system, a fragmentation problem was identified and briefly described, and some ideas for further research were suggested. An approximation technique was applied to a real-time system model for security and connectivity analysis, which avoided repetitive control (Möller, 2002). After that, a real-time property language $L_{\forall S}$ was proposed to check the rejection state of reachability and reduce safety and boundary liveness simultaneously (Aceto et al., 2003). The problems and methods in these publications have promoted the concept of exact acceleration and inspired further research. Related studies on exact acceleration in real-time model checking include Hendriks \& Larsen (2002), Yin, Song \& Zhuang (2010), Yin, Zhuang \& Wang (2011), Gou et al. (2014), Boudjadar et al. (2016), and Chadli et al. (2018). In the following four examples, the window of the acceleratable cycle is $[a, b]$.

- Hendriks \& Larsen (2002) introduced a method of syntax adjustment to a subset of timed automata by adding an appended cycle whose length was $\lceil a /(b-a)\rceil$ times longer than that of the acceleratable cycle. This method accelerates forward symbolic reachability analysis, which solves the fragmentation problem and optimizes the verification of the LEGO robotic system.

- Yin, Song \& Zhuang (2010) proposed a method to identify the acceleratable cycle in timed automata by introducing topological sorting for a large state space of a timed automaton; by simplifying the scale of timed automata, the method operated efficiently.

- An exact acceleration method based on a parking cycle was proposed (Yin, Zhuang \& Wang, 2011), in which the entry boundary condition was determined by the size of the acceleratable cycle's window (the condition is $z \geq a \times \frac{a}{b-a}+n_{0}$ ); the automaton model improved the speed of exact acceleration and reduced the cost.

- By analyzing the main parameters of the acceleration process, Gou et al. (2014) proposed a method for determining whether exact acceleration was required. This approach can be used to avoid adding an appended cycle to reduce verification speed when the number of fragments is small, or fragments do not satisfy certain conditions.

- Boudjadar et al. (2016) proposed a development method to improve the utilization rate of resources by using model-checking technology. In the design and development stage, exact acceleration technology was used to greatly improve the capability of symbolic model checking in a processing scheduling system. For the scheduling problem of network physical systems, Chadli et al. (2018) modeled advanced specifications and validation frameworks with the help 
119

120

121

122

123

124

125

126

127

128

129

130

131

132

133

134

135

136

137

138

139

140

141

142

143

144

145

146

147

148

149

150

151

152

153

154

155

156

157

of exact acceleration technology, automatically transforming high-level specifications into formal models. The above two research works mainly applied exact acceleration to model a system resource scheduling problem but did not improve the original exact acceleration theory. When modeling a complex real-time system (Wang et al., 2019), multiple acceleratable cycles may overlap at the same location. If the appended cycle method is used for exact acceleration, then the added locations multiply as the number of acceleratable cycles increases, resulting in insufficient memory for model checking. If the parking cycle method is used for exact acceleration, acceleratable-cycle stacking leads to non-uniformity in parking-cycle entry conditions; differences in the windows of multiple acceleratable cycles can increase time consumption drastically. In this paper, we propose an exact acceleration method for complex real-time model checking based on an overlapping cycle, which is an application scenario extension of parking-cycle technique. A single overlapping cycle is developed by comprehensively analyzing the accelerating effects of multiple acceleratable cycles and analyzing acceleration differences among these cycles. The overlapping cycle is simple to create and has a fixed length, eliminating the need to add multiple locations for complex real-time models. The overlapping cycle adds much less state space than appended cycles or parking cycles in model checking, substantially reducing the acceleration cost. The proposed method can be effectively applied to modeling and verification of complex real-time systems such as the IoT gateway security system. It can also alleviate additional consumption of time and space caused by state-space explosion while maintaining the original nature of the system.

The remainder of this paper is organized as follows. Section II briefly introduces timed automata, forward symbolic reachability analysis, and the theory of exact acceleration. The exact acceleration method for complex real-time models based on an overlapping cycle is proposed in Section III, which outlines the method of creating a single, fixed-length overlapping cycle. A timed automaton with an overlapping cycle is shown to accelerate the originally timed automaton with reachability. In Section IV, the acceleration effects of the appended cycle, parking cycle, and overlapping cycle with a complex real-time model example are compared using experiments. Finally, Section V provides a few ideas for future research.

\section{Preliminaries}

\section{Timed Automata}

This part is based on work by Alur \& Dill (1994). To illustrate the real-time clock of timed automata more clearly, we define a clock constraint set $T(C)$ contain all clock constraints. We assume that the set of clock variables is $C$, and the definition of the set of clock constraints $\tau$ is as follows:

$$
\tau:=\mathrm{c} \sim n \mid \tau_{1} \wedge \tau_{2}
$$

where $c \in C, \mathrm{n} \in \mathbb{N}$, and $\sim$ denotes one of the binary relationships $\{<, \leq,=, \geq,>\}$. The clock constraint set $T(C)$ is the set of all clock constraints $\tau$.

A clock interpretation $v$ is a mapping from $C$ to $\mathbb{R}^{+} \cup\{0\}$, where $\mathbb{R}^{+}$represents the set of positive real numbers. Note that $v$ assigns each clock variable in the set of clock variables $C$. For 
158

159

160

161

162

163

164

165

166

167

168

169

170

171

172

173

174

175

176

177

178

179

180

181

182

183

184

185

186

187

188

189

190

191

192

193

194

195

196

a set $X \subseteq C, X:=0$ indicates that $X$ assigns 0 to each $c \in X$ (i.e., clock reset), whereas the clock variables in set $C-X$ have no effects.

Definition 1 (Timed automaton). A timed automaton is defined as a six-tuple $\left(C, L, L_{0}, A, I, E\right)$, where $C$ is a set of clocks, $L$ is a finite set of locations, $L_{0} \subseteq L$ is the set of initial locations, $A$ is a set of action events, $I$ represents mapping that provides every location $l \in L$ with some clock constraint in $T(C)$, and $E \subseteq L \times A \times T(C) \times 2^{C} \times L$ is a set of edges. An edge $\left(l, a, \tau, \lambda, l^{\prime}\right)$ denotes a transition: when the clock constraint in location $l$ satisfies $\tau$, the system can complete action event $a$, move from location $l$ to location $l^{\prime}$, and allow clocks in $\lambda$ to be reset.

Figure 1 shows an example of a timed automaton. The timed automaton $M$ represents a plain and abstract model of the control program and the external environment in a real-time system. If the control program sends instructions to the control center in an IoT security system, the environment will be decided by sensors and actuators. The cycle of locations L1, L2, and L3 model the control program labeled the control cycle, consisting of three atomic instructions, whose clock is $x$. The external environment is modeled by clock $y$, which is checked each time in L2. The clock $y$ also called global clock. The size of the threshold constant LARGE determines how slow the environment is relative to the control program. If $y \geq$ LARGE, the control cycle may be exited.

The semantics of a timed automaton $M$ is defined by a transition system $S(M)$ with Alur \& Dill (1994). A state of $S(M)$ is a pair $(l, v)$, where $l$ is a location of $M$ and $v$ indicates a clock interpretation for $C$ such that $v$ satisfies $I(l)$. Regarding this transition system, the traces of a timed automaton have been defined by Hendriks \& Larsen (2002).

\section{Forward Symbolic Reachability Analysis}

The forward symbolic reachability analysis algorithm is a core of the real-time model-checking tool UPPAAL (Behrmann, David \& Larsen, 2004). The model-checking engine uses an on-the-fly strategy to search forward from the initial location to determine whether a symbolic state is reachable. For each symbolic state that has not yet been explored, it is necessary to calculate subsequent states based on their clocks and actions and compare them to searched symbolic states. If they have been seen in the past, they are discarded; otherwise, they are added to the list of explored symbolic states.

The reachability property $\varphi$ of a timed automaton $M$ can be presented as the timed computation tree logic (TCTL) formula $\mathrm{E}<>(P)$, where $P$ is a state property of $M$. We describe that $M$ satisfies $\varphi$, denoted by $M \vDash \varphi$, if a trace exists in the form of $\left(\left(l_{0}, v_{0}\right),\left(l_{1}, v_{1}\right), \ldots\right) \in \operatorname{Tr}(M)$, where $\left(l_{i}, v_{i}\right) \vDash P$ for some $i \geq 0$.

To describe the process of forward symbolic reachability analysis, we take automaton $M$ in Fig. 1 as an example. Table 1 shows the symbolic states that timed automaton $M$ searches forward from the initial location after one execution.

In Table 1, symbolic states 6 and 3 are both L2. However, the clock zones are not identical; these states represent two different symbolic states to be further forward searched. Therefore, every execution of the control cycle results in new symbolic states. Because the threshold LARGE is usually larger and clock $y$ is especially smaller, the timed automaton $M$ must execute a certain 
197

198

199

200

201

202

203

204

205

206

207

208

209

210

211

212

213

214

215

216

217

218

219

220

221

222

223

224

225

226

227

228

229

230

231

232

233

number of control cycles to increase clock $y$ effectively when verifying the reachability of L4. The number of executions depends on LARGE; if LARGE is large, then there are more executions. Due to different clocks, when the model checking tools detect a symbolic model cycle by cycle, many unnecessary clock fragments may appear in the state space, causing a forward symbolic fragment problem. For example, if we only observe the symbolic states 3, 6, 9 and 12 of location L2, we can find that each clock zone overlaps with the zone in front of it, which is called clock zone continuous. At this time, because of the small-time measurement in the cycle, the overlapped clock zone is divided into infinite segments, which leads to the fragmentation problem. Table 1 lists results from the UPPAAL simulator.

\section{Exact Acceleration}

Hendriks \& Larsen (2002) proposed the concept of exact acceleration, based on which, we provide basic definitions for our study. The acceleratable cycle is a key concept in exact acceleration. An acceleratable cycle can use only one clock in clock constraints (including invariants, guards, and resets).

Definition 2 (Acceleratable cycle). Let $M=\left(C, L, l_{0}, A, I, E\right)$ be a timed automaton, $E_{c}=\left(e_{0}, \ldots\right.$, $\left.e_{n-1}\right) \in E^{n}$, and $x \in C$. An acceleratable cycle is defined as a two-tuple $\left(E_{c}, x\right)$ when the following conditions are satisfied:

- $E_{c}$ is a cycle;

- for all locations in $E_{c}, I(l)$ is either empty or in the form of $\{x \leq c\}$;

- if $\left(l, a, \tau, \lambda, l^{\prime}\right) \in E_{c}$, then $\tau$ is empty or in the form of $\{x \geq c\}$, and $\lambda$ is empty or only contains $x$; and

- $x$ must be reset on all in-going edges to $\operatorname{src}\left(e_{0}\right)$.

Clock $x$ is the clock of the cycle, $I(l)$ is the location invariant, and $\tau$ is the edge guard. The location $\operatorname{src}\left(e_{0}\right)$ is the reset location whose out-going edge is $e_{0}$, which indicates the external clock's checking position in the acceleratable cycle. If a specific location's in-going edge is $e_{i}$ in the cycle, then the out-going edge of this position is $e_{i+1}$, where $i \in[0, n-2]$.

The cycle in automaton $M$ (Fig. 1), composed of locations L1, L2, and L3, is an acceleratable cycle. The clock of the cycle is $x$, and the reset location is L2. The invariants and guards are in accordance with the defined form of clock $x$, and the clock resets to zero at the only in-going edge of L2.

Definition 3 (Window of acceleratable cycle). Let an acceleratable cycle in the timed automaton $M$ be $\left(E_{c}, x\right)$. The compression sequence of all traces is expressed as $T_{r}\left(E_{c}\right)=$ $\left(\left(l_{0}, v_{0}\right),\left(l_{0}, v_{0}^{\prime}\right),\left(l_{1}, v_{1}\right), \ldots,\left(l_{n-1}, v_{n-1}\right),\left(l_{n-1}, v_{n-1}^{\prime}\right),\left(l_{n}, v_{n}\right)\right)$, where $v_{i}$ and $v_{i}^{\prime}$ indicate different clock interpretations, $i \in[0, n]$, and $l_{0}=l_{n}=\operatorname{src}\left(e_{0}\right) .\left(\left(l_{j}, v_{j}^{\prime}\right),\left(l_{j+1}, v_{j+1}\right)\right)$ depends on the edge $e_{j}$ and can be understood as an action event of $e_{j}, j \in[0, n-1]$. The window of the acceleratable cycle $\left(E_{c}, x\right)$ is defined as the interval $[a, b], a, b \in \mathbb{N}$ when the following conditions are satisfied:

- the total delay of $T_{r}\left(E_{c}\right)$ is an element of $[a, b]$; and 
234

235

236

237

238

239

240

241

242

243

244

245

246

247

248

249

250

251

252

253

254

255

256

257

258

259

260

261

262

263

264

265

266

267

268

- for any real number $d \in[a, b]$, we adjust the delays under legal clock constraints in $T_{r}\left(E_{c}\right)$ to ensure the total delay is $d$.

The meaning of the total delay in this definition is an increase in the clock of the cycle, which can be simply defined as the increment of the external clock when the acceleratable cycle returns to the reset location once from the initial location. The window is the minimal and maximal time it may take to pass through a cycle. According to this definition, the window of the acceleratable cycle shown in Fig. 1 can be calculated as $[3,7]$.

Definition 4 (Accelerated automaton based on appended cycle). Let $M=\left(C, L, l_{0}, A, I, E\right)$ be a timed automaton, and let $C y c l e=\left(E_{c}, x\right)$ be an acceleratable cycle of $M$, where $L=\left\{l_{0}, l_{1}, \ldots, l_{m}\right\}, E_{c}=$ $\left(e_{0}, e_{1}, \ldots, e_{n-1}\right), e_{i}=\left(l_{i}, a_{i}, \tau_{i}, \lambda_{i}, l_{i+1}\right)$. Acceleration of $M$ based on the appended cycle is a new automaton $\operatorname{Acc}_{a}(M, C y c l e)$ defined as $\left(C, L^{\prime}, l_{0}, A, I^{\prime}, E^{\prime}\right)$, where

- $L^{\prime}=L \cup\left\{l_{1}^{\prime}, l_{2}^{\prime}, \ldots, l_{n-1}^{\prime}\right\} \cup\left\{l_{0}^{\prime}\right\} \cup\left\{l_{1}^{\prime \prime}, l_{2}^{\prime \prime}, \ldots, l_{n-1}{ }^{\prime \prime}\right\}$

- $I^{\prime}\left(l_{i}\right)=I\left(l_{i}\right), 0 \leq i \leq m$

- $\quad I^{\prime}\left(l_{i}^{\prime}\right)=I\left(l_{i}\right), 1 \leq i \leq n-1$

- $\quad I^{\prime}\left(l_{0}^{\prime}\right)=\varnothing$

- $\quad I^{\prime}\left(l_{i}^{\prime \prime}\right)=I\left(l_{i}\right), 1 \leq i \leq n-1$

- $E^{\prime}=E \cup\left\{\left(l_{0}, a_{0}, \tau_{0}, \lambda_{0}, l_{1}^{\prime}\right),\left(l_{n-1}{ }^{\prime}, a_{n-1}, \tau_{n-1}, \lambda_{n-1}, l_{0}^{\prime}\right)\right\} \cup$

$\left\{\left(l_{0}^{\prime}, a_{0}, \tau_{0}, \lambda_{0}, l_{1}^{\prime \prime}\right),\left(l_{n-1}, a_{n-1}, \tau_{n-1}, \lambda_{n-1}, l_{0}\right)\right\} \cup$

$\left\{\left(l_{i}^{\prime}, a_{i}, \tau_{i}, \lambda_{i}, l_{i+1}^{\prime}\right),\left(l_{i}^{\prime \prime}, a_{i}, \tau_{i}, \lambda_{i}, l_{i+1}\right) \mid 1 \leq i \leq n-1\right\}$

- $\quad$ in particular, $E^{\prime}=E \cup\left\{\left(l_{0}, a_{0}, \tau_{0}, \lambda_{0}, l_{0}^{\prime}\right),\left(l_{0}^{\prime}, a_{0}, \tau_{0}, \lambda_{0}, l_{0}\right)\right\}$ when $\mathrm{n}=1$.

Theorem 1. Let $M=\left(C, L, l_{0}, A, I, E\right)$ be a timed automaton, and let Cycle $=\left(E_{c}, x\right)$ be an acceleratable cycle of $M$ with a window $[a, b]$. If $\varphi$ is the reachability property of $M$, then

$$
3 a \leq 2 b \Rightarrow\left(M \vDash \varphi \Leftrightarrow \operatorname{Acc}_{a}(M, C y c l e) \vDash \varphi\right)
$$

Theorem 1 has been proved in Hendriks \& Larsen (2002). In Definition 4, the appended cycle is obtained by expanding the acceleratable cycle twice. If the timed automaton $M$ is added to the appended cycle by expanding the acceleratable cycle $i$ times, then the precondition in Theorem 1 can be generalized to $(i+1) a \leq i b$.

Definition 5 (Accelerated automaton based on parking cycle). Let $M=\left(C, L, l_{0}, A, I, E\right)$ be a timed automaton, and let Cycle $=\left(E_{c}, x\right)$ be an acceleratable cycle of $M$ with a window of $[a, b]$, where $L$ $=\left\{l_{0}, l_{1}, \ldots, l_{m}\right\}, E_{c}=\left(e_{0}, e_{1}, \ldots, e_{n-1}\right), e_{i}=\left(l_{i}, a_{i}, \tau_{i}, \lambda_{i}, l_{i+1}\right)$. The global clock is $y$, and the maximum value of $y$ before entering the acceleratable cycle is $n_{0}$. The acceleration of $M$ based on the parking cycle is a new automaton $A c c_{p}(M, C y c l e)$ defined as $\left(C, L^{\prime}, l_{0}, A, I^{\prime}, E^{\prime}\right)$, where

- $L^{\prime}=L \cup\left\{l_{0}^{\prime}\right\}$

- $I^{\prime}\left(l_{i}\right)=I\left(l_{i}\right), 0 \leq i \leq m$

- $\quad I^{\prime}\left(l_{0}^{\prime}\right)=\varnothing$

Peer) Comput. Sci. reviewing PDF | (CS-2019:09:41765:2:0:NEW 12 Mar 2020) 


$$
\text { - } \quad E^{\prime}=E \cup\left\{\left(l_{0}, a_{0}, \tau^{\prime}, \emptyset, l_{0}^{\prime}\right),\left(l_{0}^{\prime}, a_{n-1}, \emptyset, \lambda_{n-1}, l_{0}\right)\right\}, \tau^{\prime} \text { is } y \geq a \times\left\lceil\frac{a}{b-a}\right\rceil+n_{0}
$$

Definition 5 has been given in Yin, Zhuang \& Wang (2011). The accelerated automaton $A c c_{a}$

271

$(M, C y c l e)$ equals the timed automaton $M$ with an appended cycle composed of locations $l_{0}, l_{1}^{\prime}, l_{2}^{\prime}, \ldots$,

272

$l_{n-1}{ }^{\prime}, l_{0}^{\prime}, l_{1}^{\prime}, l_{2}^{\prime \prime}, \ldots, l_{n-1}$. Accelerated automaton $A c c_{p}(M, C y c l e)$ equals the timed automaton $M$ with a

273

parking cycle whose edge guard $y$ controls the acceleration timing. Only when the acceleratable

274

275 cycle has been executed at least $[a /(b-a)]$ times is the automaton permitted to enter the parking

276 cycle. Figures 2 and 3 display the acceleration of $M$ (Fig. 1) based on the appended cycle and parking cycle, respectively. They are labeled the accelerated automata $M_{a}$ and $M_{p}$. Because the

277 window of the acceleratable cycle is $[3,7]$, the edge guard of the parking cycle is $y \geq 3$ in $M_{p}$.

Theorem 2. Let $M=\left(C, L, l_{0}, A, I, E\right)$ be a timed automaton, and let Cycle $=\left(E_{c}, x\right)$ be an acceleratable cycle of $M$ with a window of $[a, b]$. If $\varphi$ is a reachability property of $M$, then

280

$$
a<b \Rightarrow\left(M \vDash \varphi \Leftrightarrow \operatorname{Acc}_{p}(M, C y c l e) \vDash \varphi\right)
$$

Yin, Zhuang \& Wang (2011) gives this theorem form forward symbolic reachability analysis, and this is the previous achievement of our working group. We will give its another proof in the view of zone later.

284

\section{Exact Acceleration of Complex Real-time System Model Based on}

\section{Overlapping Cycle}

287

288

289

290

291

292

293

294

295

296

297

298

299

300

301

302

303

304

305

306

The appended cycle and parking cycle technologies in exact acceleration apply to a real-time system model with a single acceleratable cycle. For a complex real-time system model (as shown in Fig. 4), using these two technologies for exact acceleration requires additional states and consumes a large amount of time cost, which influences the acceleration effect.

Figure 4 presents an example of the IoT gateway security system (in Wang et al., 2018). The timed automaton $M^{\prime}$ models a wireless sensor network including a reactive program and external environment. The run-time behavior control several sensors, which can be transformed into clock constrains in UPPAAL. Every location in the cycle represents a sensor model in the IoT system. The cycle's clock is $x$, and clock $y$ controls the execution time. The larger the constant LARGE is, the more slowly the timed automaton $M^{\prime}$ runs. Using the algorithm described by Yin, Song \& Zhuang (2010) to identify the acceleratable cycle in $M^{\prime}$, we obtain four acceleratable cycles whose reset locations are all L1 and share the clock of cycle $x$. For this complex real-time model, we propose a method based on the overlapping cycle for exact acceleration.

Theorem 3. Let $M=\left(C, L, l_{0}, A, I, E\right)$ be a timed automaton, and let Cycle $=\left(E_{c}, x\right)$ be an acceleratable cycle of $M$ with a window of $[a, b]$. If $a<b$, there is a positive integer $n$ in the forward symbolic reachability analysis, which leads the reset location to obtain a continuous clock zone after executing the Cycle $n$ times.

Proof. According to the forward symbolic reachability analysis, the problem of fragments in the acceleratable cycle will inevitably lead to the overlap of clock zones, that is, the appearance of continuous clock zone. If $a<b$, according to the definitions about exact acceleration, the 
307

308

309

310

311

312

313

314

315

316

317

318

319

320

321

322

323

324

325

326

327

328

329

330

331

332

333

334

335

336

337

338

339

340

341

342

343

344

continuous clock zone will be got after several executions of the acceleratable cycle, and the point of the proof is to determine the number of executions.

So, without loss of generality, we might assume that the execution number is a positive integer $n$. Let $n$ be the rounds of $C y c l e$ execution, and let the interval $[c, d]$ be the clock zone at the reset location before execution of the Cycle. At the reset location, the clock zone is continuous from the $(n+1)$ th time onward; therefore, the clock zones obtained in the $(n+1)$ th time and the $n$th time have an intersection that is

$$
(n+1) a+c \leq n b+d \Rightarrow n \geq(a+c-d) /(b-a)
$$

Because $b>a, d \geq c$, there must be an integer $n \geq a /(b-a)$. So, the number of executions should be at least $[a /(b-a)]$. When the Cycle is executed $[a /(b-a)\rceil$ (that is $n)$ times, the reset location obtains a continuous clock zone, thereby completing the proof.

Corollary 1 . If the timed automaton $M$ has an acceleratable cycle with a window of $[a, b], a<b$, then the reset location will obtain a continuous clock zone after executing the Cycle at least $a /(b-a)$ times during forward symbolic reachability analysis.

Proof. According to Theorem 3, we easily know the reset location obtains a continuous clock zone when the Cycle is executed $n$ times. For the integer $n$, we can calculate that $n \geq a /(b-a)$. The proof is completed.

Corollary 2. Let the global clock be $y$, and let the maximum value of $y$ before entering the acceleratable cycle be $n_{0}$. If the timed automaton $M$ has an acceleratable cycle with a window of $[a, b], a<b$, then the reset location will obtain a continuous clock zone when the following condition is satisfied during forward symbolic reachability analysis:

$$
y-n_{0} \geq a \times a /(b-a)
$$

Proof. According to Corollary 1, the reset location will obtain a continuous clock zone after executing the acceleratable cycle at least $a /(b-a)$ times. Because the window of the acceleratable cycle is $[a, b]$, the increment of $y$ by executing the acceleratable cycle $a /(b-a)$ times is denoted as $\Delta y \in[a \times a /(b-a), b \times a /(b-a)]$, where $\Delta y=y-n_{0}$. Therefore, when $y-n_{0} \geq a \times a /(b-$ $a)$, the reset location will obtain a continuous clock zone. The proof is completed.

Corollary 3. Let the global clock be $y$, and let the maximum value of $y$ before entering the acceleratable cycle be $n_{0}$. If the timed automaton $M$ has an acceleratable cycle with a window of $[a, b], a<b$, then every location in the acceleratable cycle will obtain a continuous clock zone when $y-n_{0} \geq a \times a /(b-a)$.

Proof. Because clock $y$ is not the cycle clock, any invariant or guard in the acceleratable cycle will not contain clock $y$ according to Definition 2. Based on the theory of timed automata, clock $y$ exhibits monotonous growth when the acceleratable cycle is executed. Thus, when $y-n_{0} \geq$ $a \times a /(b-a)$, per Corollary 2 , the reset location begins to obtain a continuous clock zone, 
345

346

347

348

349

350

351

352

353

354

355

356

357

358

359

360

361

362

363

364

365

366

367

368

369

370

371

372

373

374

375

376

377

378

379

380

indicating that every location in the acceleratable cycle is reachable. At this point, a constant clock zone will also be received by any location in the acceleratable cycle, and the proof is completed.

Next, we will give the new proof of Theorem 2.

Proof. Sufficient Condition. The known condition is $a<b$. Because $A c c_{p}(M, C y c l e)$ is obtained by adding a parking cycle to $M$, the timed automaton $M$ can clearly reach any reachable state in original model. The accelerated automaton $\operatorname{Acc}_{p}(M, C y c l e)$ can also reach states by executing the same time trace; that is, the state transition system $S(M)$ associated with $M$ is included in the state transition system $S\left(A c c_{p}(M, C y c l e)\right)$ associated with $A c c_{p}(M, C y c l e)$.

Necessary Condition. The known condition is $a<b$. Let the global clock be $y$. When $y<a \times a$ $/(b-a)+n_{0}$, the accelerated automaton $A c c_{p}(M, C y c l e)$ does not execute the parking cycle, and reachable states in $\operatorname{Acc}_{p}(M, C y c l e)$ are also reachable in the timed automaton $M$. When $y \geq a \times a$ $/(b-a)+n_{0}$ (according to Corollary 3), every location in the acceleratable cycle of $M$ will obtain a continuous clock zone; that is, after $y \geq a \times a /(b-a)+n_{0}$ at any time, $M$ can reach any location in the acceleratable cycle and $\operatorname{Acc}_{p}(M, C y c l e)$ executes the parking cycle, satisfies the edge guards, and returns to the reset location of any state, which guarantees that $M$ is always reachable.

In summary, when $a<b$, the accelerated automaton $A c c_{p}(M, C y c l e)$ does not change the reachability property $\varphi$ of the timed automaton $M$, and the proof is completed.

According to our theorems and corollaries, we can prove that the exact acceleration method based on the parking cycle is more concise and effective than that based on the appended cycle. On one hand, there is no location invariant in the parking cycle to ensure the clock can stay in this location for acceleration; on the other hand, the parking cycle contains an edge guard to ensure that any location of the acceleratable cycle obtains a continuous clock zone, which provides reachability. In this way, the parking cycle accelerates the search for the symbolic state by controlling acceleration timing and ensures reachability of the timed automaton to realize exact acceleration. The exact acceleration method for the complex real-time model based on an overlapping cycle is an improved method based on the parking cycle. It attempts to extend the application field of exact acceleration technology to complex real-time model checking to improve efficiency and alleviate state explosion.

Theorem 4. Let $M=\left(C, L, l_{0}, A, I, E\right)$ be a timed automaton with several acceleratable cycles. Let Cycle $_{i}=\left(E_{c_{i}} x\right)$ be the $i$ th acceleratable cycle of $M$ with a window of $\left[a_{i}, b_{i}\right]$, where $i$ is a non-zero natural number. All acceleratable cycles affect the cycle of clock $x$, and their reset locations are uniform in $l_{\text {reset }} \in L$. There is a single acceleratable cycle whose effect is the most effective in obtaining a continuous clock zone than multiple acceleratable cycles. 
381 Proof. Let $n_{j}=\left\lceil\frac{a_{j}}{b_{j}-a_{j}}\right\rceil \times a_{j}, n_{k}=\left\lceil\frac{a_{k}}{b_{k}-a_{k}}\right\rceil \times a_{k}$, where $1 \leq j, k \leq i$, and $j, k$ are non-zero natural 382 numbers. Then, $n_{j}$ and $n_{k}$ represent the edge guard of the $j$ th and $k$ th acceleratable cycle, 383 respectively, when adding a parking cycle. If these two acceleratable cycles are executed 384 simultaneously, the edge guard can be expressed as $n_{j k}=\left[\frac{a_{j}+a_{k}}{\left(b_{j}-a_{j}\right)+\left(b_{k}-a_{k}\right)}\right] \times\left(a_{j}+a_{k}\right)$. The 385 window of the $i$ th acceleratable cycle is $\left[a_{i}, b_{i}\right]$ as a known condition, where $0 \leq a_{j} \leq b_{j}, 0 \leq a_{k} \leq$ $386 b_{k}$, and there is $a_{j}+a_{k} \geq a_{j}, a_{k}$.

$387 \quad$ We make $\frac{a_{j}}{b_{j}-a_{j}}=\frac{U}{V}, \frac{a_{k}}{b_{k}-a_{k}}=\frac{X}{Y}$, such that $\frac{a_{j}+a_{k}}{\left(b_{j}-a_{j}\right)+\left(b_{k}-a_{k}\right)}=\frac{U+X}{V+Y}$. We assume that $\frac{U}{V}$ is smaller, 388 then $\frac{X}{Y}=\frac{U+\mu}{V}, \mu \in \mathbb{R}^{+}$. Therefore, there is $\frac{U+X}{V+Y}=\frac{U+U+\mu}{V+V}=\frac{U}{V}+\frac{\mu}{2 V}>\frac{U}{V}$; that is, $\left\lceil\frac{U+X}{V+Y}\right\rceil \geq\left\lceil\frac{U}{V}\right\rceil$ and 389 $\left.\left\lceil\frac{U+X}{V+Y}\right\rceil \geq \min \left(\left\lceil\frac{U}{V}\right\rceil, \mid \frac{X}{Y}\right\rceil\right)$. In the positive-number condition, a larger number multiplied by a larger number is either equal to or greater than a smaller number multiplied by a smaller number; therefore,

which is $n_{j k} \geq \min \left(n_{j}, n_{k}\right)$.

According to Corollary 1 , the reset location will obtain a continuous clock zone after executing the acceleratable cycle, which has a smaller value of $\left[\frac{a_{i}}{b_{i}-a_{i}}\right] \times a_{i},\left[\frac{a_{i}}{b_{i}-a_{i}}\right]$ times during forward symbolic reachability analysis. This solution is faster than using two acceleratable cycles simultaneously to obtain a continuous clock zone, and it is better than using the larger one.

By extension, when comparing any two acceleratable cycles, a shorter time cycle always obtains a continuous clock zone more quickly. When comparing all acceleratable cycles, we can achieve the most effective acceleratable cycle for exact acceleration. This result indicates that the acceleration effect of a single acceleratable cycle is more effective than that of multiple 403 acceleratable cycles, thereby completing the proof.

Corollary 4. Let $M=\left(C, L, l_{0}, A, I, E\right)$ be a timed automaton with several acceleratable cycles. Let Cycle $e_{i}=\left(E_{c_{i}}, x\right)$ be the $i$ th acceleratable cycle of $M$ with a window of $\left[a_{i}, b_{i}\right]$, where $i$ is a non-zero 406 natural number. All acceleratable cycles affect the cycle of clock $x$, and their reset locations are 407 uniform in $l_{\text {reset }} \in L$. If $a_{i}<b_{i}$, then the acceleratable cycle with the $\min \left(\left[\frac{a_{i}}{b_{i}-a_{i}}\right] \times a_{i}\right)$ has the best acceleration effect of obtaining a continuous clock zone in the shortest time.

Proof. According to Theorem 4, comparing any two acceleratable cycles, a cycle with smaller value of $\left(\left[\frac{a_{i}}{b_{i}-a_{i}}\right] \times a_{i}\right)$ always obtains a continuous clock zone more quickly. When comparing all 
411 acceleratable cycles, the cycle with the $\min \left(\left[\frac{a_{i}}{b_{i}-a_{i}}\right] \times a_{i}\right)$ obviously has the most effective 412 acceleration. The proof is completed.

413

414 Definition 6 (Accelerated automaton based on overlapping cycle). Let $M=\left(C, L, l_{0}, A, I, E\right)$ be a 415 timed automaton with $k$ acceleratable cycles, where $L=\left\{l_{0}, l_{1}, \ldots, l_{m}\right\} . C Y C L E=\left\{\right.$ Cycle $_{1}, \ldots, C y c l e_{k}$ $\left.416 \mid k \in \mathbb{N}^{+}\right\}$denotes the acceleratable cycle set. Let $C y c l e_{i}=\left(E_{c_{i}}, x\right)$ be the $i$ th acceleratable cycle 417 with a window of $\left[a_{i}, b_{i}\right]$, where $0 \leq i \leq k, E_{c}=\left(e_{0}, e_{1}, \ldots, e_{n-1}\right), e_{j_{i}}=\left(l_{j_{i}}, a_{j_{i}}, \tau_{j_{i}}, \lambda_{j_{i}}, l_{(j+1)}\right)$. All 418 acceleratable cycles affect the cycle of clock $x$, and their reset locations are uniform in $l_{\text {reset }} \in L$. 419 The global clock is $y$, and the maximum value of $y$ before entering the acceleratable cycle is $n_{0}$. 420 The acceleration of $M$ based on the overlapping cycle is a new automaton $\operatorname{Acc}_{o}(M, C Y C L E)$ defined 421 as $\left(C, L^{\prime}, l_{0}, A, I^{\prime}, E^{\prime}\right)$, where

422

423

- $L^{\prime}=L \cup\left\{l_{\text {reset }}{ }^{\prime}\right\}$

- $I^{\prime}\left(l_{h}\right)=I\left(l_{h}\right), 0 \leq h \leq m$

- $\quad I^{\prime}\left(l_{\text {reset }}^{\prime}\right)=\varnothing$

- $E^{\prime}=E \cup\left\{\left(l_{\text {reset }}, \emptyset, \tau^{\prime}, \emptyset, l_{\text {reset }}{ }^{\prime}\right),\left(l_{\text {reset }}{ }^{\prime}, \emptyset, \emptyset, \lambda^{\prime}, l_{\text {reset }}\right)\right\}, \tau^{\prime}$ is $y \geq \min \left(\left[\frac{a_{i}}{b_{i}-a_{i}}\right] \times a_{i}\right)+n_{0}$ and $\lambda^{\prime}$ only contains $x$

The accelerated automaton $A c c_{o}(M, C Y C L E)$ equals the timed automaton $M$ with the addition of an overlapping cycle at only one reset location, which solves the problem where an exact acceleration cannot be used directly for complex real-time model checking. The overlapping cycle only needs to analyze all windows of every acceleratable cycle in CYCLE for the calculation. We can also avoid using an appended cycle or parking cycle for each acceleratable cycle, greatly reducing the additive symbolic state. Figure 5 depicts the acceleration of $M^{\prime}$ (Fig. 4) based on an overlapping cycle, named accelerated automaton $M_{O}^{\prime}$. Because the timed automaton $M^{\prime}$ contains four acceleratable cycles, we analyze them separately, discard the deadlocked cycle, and only retain three executable cycles. The deadlocked cycle consists with location $l_{1}, l_{2}, l_{3}, l_{4}, l_{5}, l_{6}, l_{7}$ , $l_{8}, l_{9}, l_{10}, l_{14}, l_{13}, l_{1}$ in sequence. For further analysis, the windows of acceleratable cycles are calculated as $[7,18],[6,16]$, and $[13,24]$. By taking the minimum value of $\left\lceil\frac{a_{i}}{b_{i}-a_{i}}\right\rceil \times a_{i}$, we can obtain the overlapping cycle's entry condition, which is $y \geq 6$.

Theorem 5. Let $M=\left(C, L, l_{0}, A, I, E\right)$ be a timed automaton with several acceleratable cycles. Let Cycle $_{i}=\left(E_{c_{i}}, x\right)$ be the $i$ th acceleratable cycle of $M$ with a window of $\left[a_{i}, b_{i}\right]$, where $i$ is a nonzero natural number. If $x$ is reset on edge $e_{0}$, then the subsequent states of $\operatorname{src}\left(e_{0}\right)$ reachable by multiple acceleratable cycles in $M$, are reachable by exactly one execution of the overlapping cycle in $A C c_{o}(M, C Y C L E)$. 
444

445

446

447

448

449

450

451

452

453

454

455

456

457

458

459

460

461

462

463

464

465

466

467

468

469

470

471

472

473

474

475

476

477

478

479

480

481

482

Proof. For a certain acceleratable cycle, its window is set as $\left[a^{\prime}, b^{\prime}\right]$. According to Theorem 1, when $3 a^{\prime} \leq 2 b^{\prime}$, the appended cycle does not change the subsequent reachability of the reset location $\operatorname{src}\left(e_{0}\right)$ in $M$. According to Theorem 2, when $a^{\prime} \leq b^{\prime}$, the parking cycle does not change the subsequent reachability of the reset location $\operatorname{src}\left(e_{0}\right)$ in $M$. In the case of multiple acceleratable cycles superimposing on the same location in a complex real-time system, the subsequent reachability of the reset location $\operatorname{src}\left(e_{0}\right)$ in $M$ can be guaranteed to remain unchanged if any part of the acceleratable cycle is processed with exact acceleration.

According to Theorem 4, the accelerable cycle is more effective for obtaining a continuous clock zone at reset location $\operatorname{src}\left(e_{0}\right)$ than multiple acceleratable cycles. In particular, according to Corollary 4 , if $a_{i}<b_{i}$, then the exact acceleration based on overlapping cycle can obtain the continuous clock zone in the shortest time. The proof is completed.

This theorem ensures the effectiveness of acceleration. For a single acceleratable cycle, if all states are reachable by more than one execution of the acceleratable cycle, then exactly only one execution of the acceleratable cycle of the appended cycle or parking cycle can guarantee reachability of all states in the accelerative automaton. The complex real-time model checking differs from the exact acceleration of a single acceleratable cycle. In depth-first forward symbolic reachability analysis, it is necessary to verify whether subsequent states are reachable in priority while ignoring the breadth-first search within cycles.

In our case study of an IoT gateway security system (Wang et al., 2018), the control center must complete a security process and distribute it to each sensor node. Once a self-organizing sensor network completes the process, it can respond to the command of the control center in a timely manner. The control center can perform subsequent operations after receiving feedback regardless of whether other sensor nodes can complete the process. Hence, the security system must ensure its subsequent reachability regardless of who completes the process. This approach accelerates the search of subsequent states, thus avoiding time and space consumption caused by superposition of acceleratable cycles.

\section{Experimental Results}

To verify the validity of the exact acceleration method based on an overlapping cycle for complex real-time model checking, we collected runtime data, including memory consumption and verification time, from the timed automaton $M^{\prime}$. We also gathered runtime data from the accelerated automata $M_{a}^{\prime}, M_{p}^{\prime}$, and $M_{o}^{\prime}$, which use the appended cycle, parking cycle, and overlapping cycle, respectively. We employed the model-checking tool UPPAAL with a depthfirst search order to verify whether location L15 was reachable, which can give the time and memory consumption in verification automatically. Experimental results are displayed in Table 2.

Results show that the time consumption of $M^{\prime}$ increased with exponential growth of LARGE at a rate of nearly ten times without using exact acceleration. The memory consumption of $M^{\prime}$ increased slightly because no additional locations were added. The accelerated automaton $M_{a}^{\prime}$ used 
483 an appended cycle, which reduced the time consumption, reflecting the advantages of exact 484 acceleration. However, due to a large number of additional locations, the memory consumption of $485 M_{a}^{\prime}$ increased dramatically. The accelerated automaton $M_{p}^{\prime}$ used a parking cycle to reduce the time 486 consumption further compared to $M_{a}^{\prime}$. The fixed length of the parking cycle reduced the number 487 of additional locations compared to the appended cycle; accordingly, the memory consumption 488 was much lower for $M_{p}^{\prime}$ than for $M_{a}^{\prime}$ but slightly higher than for $M^{\prime}$. The accelerated automaton $M_{o}^{\prime}$ 489 that used the proposed overlapping cycle exhibited minimal time consumption and only required 490 the addition of a single, fixed-length location for the complex real-time model. The memory 491 consumption of $M_{o}^{\prime}$ was close to that of $M^{\prime}$, far less than that of $M_{a}^{\prime}$, and slightly better than that of 492 $M_{p}^{\prime}$. We can explain the time and memory consumption of $M_{o}^{\prime}$ by Theorem 5 . The depth-first search order ensures that the overlapping cycle accelerates exploration before complete exploration of all accelerable cycles.

A less theoretical study case involves model verification of the IoT gateway security system (Wang et al., 2018). The exact acceleration method based on the overlapping cycle was successfully applied in this case, significantly improving verification efficiency. The technical framework of the IoT gateway security system is illustrated in Fig. 6.

An essential technology in the IoT gateway security system is the time-stamped advanced encryption standard algorithm. By introducing a timestamp into the key expansion phase, the round key can be dynamically updated with change over time to realize a cipher text change that ensures the security of confidential information. Due to the introduction of a timestamp, the system generates acceleratable cycles when modeled as timed automata. Multiple acceleratable cycles are overlaid on the same location in particular scenarios, which requires overlapping cycle technology for exact acceleration.

Our theory can be used to simulated the parallel execution of processes and idle cycles. However, the presence of urgent locations and synchronous channels may disturb exact acceleration. For example, if broadcast channel coordination occurs in an urgent location, the multi-party response of the broadcast should be completed before the next state location can be migrated. The execution time of the response process is not controlled by the cycle control program; thus, it is not appropriate to simply use exact acceleration technology for acceleration; the space-time loss of using acceleration technology should be compared to the broadcasting response. However, exact acceleration technology can often handle urgent locations and synchronous channels. The following case of an IoT gateway security system also involves these situations. As no extra time interference exists within the whole acceleration process, the exact acceleration technology can finally be successfully applied to system modeling and verification.

517 The accelerated automaton based on an overlapping cycle is an approximation that can be 518 adapted to verify the accuracy of invariance and reachability properties. Consider the processes in 519 Fig. 7; these processes model a top architecture and a middleware control program consisting of 520 locations, edges, and channels. 
521

522

523

524

525

526

527

528

529

530

531

532

533

534

535

536

537

538

539

540

541

542

543

544

545

546

547

548

549

550

551

552

553

554

555

556

557

558

559

560

The IoT gateway runs from the Start location, reads configuration information and performs gateway identity authentication. The underlying unified authentication service is invoked through channel StartAC for security authentication, and GatewayStatus is returned after authentication. If GatewayStatus=true, then the system enters the location EnterMiddle and transforms to the polling module of the middle layer through the StartMiddle channel. For the middle-layer polling module, polling begins through the StartMiddle channel, and the top-layer main module is returned by the FinishMiddle channel. Location CheckCategory controls whether polling logic ends up at a perception terminal or an execution terminal, which each have different processing methods. In the two polling processes, the underlying security service is invoked through the synchronization channel according to different requirements. The specific process can be interpreted by the meaning of state locations, synchronization channels, and variables as described by Wang et al. (2018). In particular, clock constraints are added during the stage of waiting for timing and the stage of keeping the equipment running. The top-layer main module model and middle-layer polling module model constitute the general framework of the IoT gateway security system. Security implementation depends on the underlying security service modules ultimately, hence it is necessary to improve model construction of the underlying security service modules. For complex system modeling with underlying services, we apply the exact acceleration method proposed in this paper, which can effectively improve the verification speed.

To demonstrate the effect of exact acceleration, we checked all security properties of the IoT gateway security system model in Fig. 7. Several examples are presented below.

(1) A[] not deadlock

Property 1 is used to check deadlock and ensure all state locations will be reachable.

(2) $\mathrm{E}<>$ Top.CheckGS

(3) E $<>$ Top.EnterMiddle imply Middle.CheckCategory

Properties 2 and 3 are used to explore part of the state space. The truth of these two properties indicates that the implementation accelerated model is an exact acceleration with the overlapping cycle.

(4) A[] Top.Restart imply $c<=300$

(5) A[] Top.Record imply $c<=600$

(6) A[] Middle.RetrieveData imply Middle. $y>=30$

(7) A[] Middle.WaitDevice imply Middle. $y<=5$

Properties 4-7 are examined in terms of whether subsequent states of the reset location are reachable. Clock $c$ is a global clock and clock $y$ is used to model the duration of one process.

We measured time and memory consumption and explored states for these properties. The IoT gateway security system was modeled as a timed automaton $M_{I o T}$, and the acceleration of $M_{I o T}$ with overlapping cycles was modeled as an automaton $M_{I o T}$. We used model checkers UPPAAL and KRONOS to verify security system properties automatically, such as confidentiality, availability, and authenticity in parallel processes. KRONOS is able to complete the statistics of the state scale traversed by the whole verification process. It makes up for the fact that UPPAAL can't do this. Table 3 lists the experimental results. 
561

562

563

564

565

566

567

568

569

570

571

572

573

574

575

576

577

578

579

580

581

582

583

584

585

586

587

588

589

590

591

\section{2}

593

594

595

596

597

598

599

600

On the premise of guaranteeing the security of IoT gateway system, a large number of underlying services and various applications can be embedded in the system framework. At this time, the security requirements of IoT gateway system are mainly for various new access services, and the framework security of the gateway itself can be maintained by its own mechanism. After access to a large number of services and applications, the original model will become complex, concurrent, real-time with large-scale. The verification of the system needs to be processed by the exact acceleration method based on overlapping cycle.

With the increase of the number of access services, the system model becomes more and more complex, and the scale of access number greatly affect the efficiency of model verification. Appended cycle and parking cycle methods are more suitable for single accelerating cycle scenarios. In this complex scenario, when the number of services reaches a certain level, the acceleration process may not be completed. According to the change of the number of access services, Table 4 gives the comparison of the acceleration effects of different exact acceleration methods (from the perspective of time).

The results show that for complex real-time systems, the acceleration efficiency of overlapping cycle is much higher than that of appended cycle and parking cycle, and the verification can still be completed when the state scale reaches $10^{7}$ with proposed method. So, the exact accelerating technology substantially reduced the time required for verification in complex real-time model checking. Overlapping cycle acceleration demonstrated the highest efficiency compared to the appended cycle and parking cycle. In the simple example of automaton $M$ ' in Fig. 4, 55 additional locations were required when using the appended cycle, much higher than the number of locations in the original model. Although the appended cycle reduced verification time, it increased the difficulty of adding locations to the model in an early stage. When many acceleratable cycles were stacked in the same reset position, more than one location needed to be added to $M^{\prime}$ when the parking cycle was used, although the length of the parking cycle was fixed. The parking cycle was neither simpler nor faster than the overlapping cycle, and its previous calculation was larger than that of the overlapping cycle.

With the exception of this IoT case, our approach can be applied to other scenarios, such as security validation of blockchain smart contracts. The complete code and UPPAAL model can be found at https://github.com/iegqwang/UPPAAL.

\section{Conclusions}

To solve the fragmentation problem for complex real-time model checking, we propose an exact acceleration method based on an overlapping cycle, which is an application scenario extension of parking-cycle technique, to accelerate forward symbolic reachability analysis. Compared with the appended cycle or parking cycle for exact acceleration, the proposed method can be applied to the model acceleration of large-scale complex real-time systems and only requires the addition of a single, fixed-length location to the system's timed automaton model. The addition of an overlapping cycle introduces far fewer symbolic states than using either an appended cycle or parking cycle. Rather than relying on windows of acceleratable cycles, the proposed accelerated

Peer) Comput. Sci. reviewing PDF | (CS-2019:09:41765:2:0:NEW 12 Mar 2020) 
601 automaton model is more straightforward and reduces the space-time overhead of exact 602 acceleration.

603 Two aspects warrant exploration in future research. First, we must continue to study the 604 algorithm for the acceleratable cycle, try to simplify the original automaton model, guarantee its 605 original property, and rapidly identify the deadlock. Second, we plan to develop a simple exact 606 acceleration automatic checking platform that can consider other practical conditions such as 607 action transitions, urgent locations, and synchronous channels to solve actual modeling problems 608 more efficiently.

609

\section{References}

611 Aceto L, Bouyer P, Burgueño A, Larsen KG. 2003. The power of reachability testing for

612 timed automata. Theoretical Computer Science 300(1):411-475 DOI 10.1016/S0304-

613 3975(02)00334-1.

614 Alur R, Dill DL. 1994. A theory of timed automata. Theoretical Computer Science 126(2):183-

615235 DOI 10.1016/0304-3975(94)90010-8.

616 Barnat J, Bauch P, Brim L, Češka M. 2010. Employing multiple CUDA devices to accelerate

617 LTL model checking. In: International Conference on Parallel and Distributed Systems, 2010.

618 Piscataway: IEEE, 259-266 DOI 10.1109/ICPADS.2010.82.

619 Behrmann G, Bouyer P, Larsen KG, Pelánek R. 2006. Lower and upper bounds in zone-based 620 abstractions of timed automata. International Journal on Software Tools for Technology Transfer 621 8(3):204-215 DOI 10.1007/s10009-005-0190-0.

622 Behrmann G, David A, Larsen KG. 2004. A tutorial on Uppaal. In: International School on 623 Forman Methods for the Design of Computer, Communication and Software Systems, 2004.

624 Berlin: Springer Verlag, 200-236 DOI 10.1007/978-3-540-30080-9_7.

625 Boudjadar A, David A, Kim JH, Larsen KG, Mikučionis M, Nyman U, Skou A. 2016.

626 Statistical and exact schedulability analysis of hierarchical scheduling systems. Science of

627 Computer Programming 127: 103-130 DOI 10.1016/j.scico.2016.05.008.

628 Chadli M, Kim JH, Larsen KG, Legay A, Naujokat S, Steffen B, Traonouez LM. 2018.

629 High-level frameworks for the specification and verification of scheduling problems.

630 International Journal on Software Tools for Technology Transfer 20(4):397-422 DOI

631 10.1007/s10009-017-0466-1.

632 Chen H, Cui L. 2016. Design and model checking of service-oriented software architecture for 633 Internet of things: A survey. Chinese Journal of Computers 39(5):853-871 DOI 634 10.11897/SP.J.1016.2016.00853.

635 Cruz JP, Kaji Y, Yanai N. 2018. RBAC-SC: Role-based access control using smart contract. 636 IEEE Access 6:12240-12251 DOI 10.1109/ACCESS.2018.2812844.

637 Dubout C, Fleuret F. 2013. Accelerated training of linear object detectors. In: Computer Vision 638 and Pattern Recognition Workshops, 2013. Piscataway: IEEE, 572-577 DOI

639 10.1109/CVPRW.2013.156. 
640 Gou L, Li Z, Wang C, Zhuang L. 2014. A method to determine the exact acceleration 641 efficiency in model checking. Journal of Zhongyuan University of Technology 25(4):37-41 DOI 642 10.3969/j.issn.1671-6906.2014.04.009.

643 Grishchenko I, Maffei M, Schneidewind C. 2018. Foundations and tools for the static analysis 644 of ethereum smart contracts. In: Computer Aided Verification, 2018. Berlin: Springer Verlag, 5164578 DOI 10.1007/978-3-319-96145-3_4.

646 Han D, Yang Q, Xing J. 2015. UML-based modeling and formal verification for software self647 adaptation. Journal of Software 26(4):730-746 DOI 10.13328/j.cnki.jos.004758.

648 Hendriks M, Larsen KG. 2002. Exact acceleration of real-time model checking. Electronic 649 Notes in Theoretical Computer Science 65(6):120-139 DOI 10.1016/S1571-0661(04)80473-0.

650 Herbreteau F, Srivathsan B, Walukiewicz L. 2016. Better abstractions for timed automata. 651 Information and Computation 251:67-90 DOI 10.1016/j.ic.2016.07.004.

652 Iversen T K, Kristoffersen K J, Larsen K G, Laursen M, Madsen R G, Mortensen S K, 653 Pettersson P, Thomasen C B. 2000. Model-checking real-time control programs: Verifying

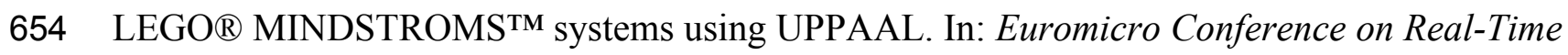
655 Systems, 2000. Piscataway: IEEE, 147-155 DOI 10.1109/EMRTS.2000.854002.

656 Jeong H, Yoo Y, Yi K M, Choi J Y. 2014. Two-stage online inference model for traffic pattern 657 analysis and anomaly detection. Machine Vision and Applications 25(6):1501-1517 DOI 658 10.1007/s00138-014-0629-y.

659 Konur S, Fisher M, Schewe S. 2013. Combined model checking for temporal, probabilistic, and 660 real-time logics. Theoretical Computer Science 503:61-88 DOI 10.1016/j.tcs.2013.07.012.

661 Lee J, Yu S, Park K, Y Park, Park Y. 2019. Secure three-factor authentication protocol for 662 multi-gateway IoT environments. Sensors 19(10):2358-2383 DOI 10.3390/s19102358.

663 Li G, Wei Q, Li L, Jin Z, Xu Y, Zheng L. 2013. Environment based modeling approach for 664 services in the Internet of things. Science China Press, 43(10):1198-1218 DOI 665 10.1360/N112013-00031.

666 Lin K, Chen Y, Xu D. 2017. Reliability assessment model considering heterogeneous 667 population in a multiple stresses accelerated test. Reliability Engineering \& System Safety 668 165:134-143 DOI 10.1016/j.ress.2017.03.013.

669 Möller MO. 2002. Parking can get you there faster: Model augmentation to speed up real-time 670 model checking. Electronic Notes in Theoretical Computer Science 65(6):202-217 DOI 671 10.1016/S1571-0661(04)80477-8.

672 Pinisetty S, Jéron T, Tripakis S, Falcone Y, Marchand H, Preoteasa V. 2017. Predictive 673 runtime verification of timed properties. Journal of Systems and Software 132:353-365 DOI 674 10.1016/j.jss.2017.06.060.

675 Qanadilo M, Samara S, Zhao Y. 2013.c. In: Latin-American Symposium on Dependable 676 Computing, 2013. Piscataway: IEEE, 40-47 DOI 10.1109/LADC.2013.20.

677 Wang C, Pastore F, Briand L. 2019. Oracles for testing software timeliness with uncertainty. 678 ACM Transactions on Software Engineering and Methodology 8(1):1-30 DOI 10.1145/3280987. 
679 Wang G, Zhuang L, Wang R, Song Y, Zhang K. 2018. Modeling and verifying based on

680 timed automata of Internet of things gateway security system. Journal on Communications

681 39(3):63-75 DOI 10.11959/j.issn.1000-436x.2018042.

682 Wang H, Zhong D, Zhao T, Ren F. 2019. Integrating model checking with SysML in complex

683 system safety analysis. IEEE Access 7:16561-16571 DOI 10.1109/ACCESS.2019.2892745.

684 Yin C, Song W, Zhuang L. 2010. Method of acceleratable cycles in identify timed automata.

685 Computer Engineering and Design 31(23):5113-5115 DOI 10.16208/j.issn1000-

6867024.2010 .23 .030$.

687 Yin C, Zhuang L, Wang C. 2011. Exact acceleration of real-time model checking based on

688 parking cycle. Acta Electronica Sinica 39(3):489-493 DOI 10.3969/j.issn.0372-

689 2112.2011.03.001.

690 Zhang F, Bu L, Wang L, Zhao J, Li X. 2013. Modeling and analysis of wireless sensor

691 network protocols by stochastic timed automata and statistical model checking. Scientia Sinica

692 Informationis 43(1):90-107 DOI 10.1360/112012-498. 
Figure 1

Timed automaton $M$.

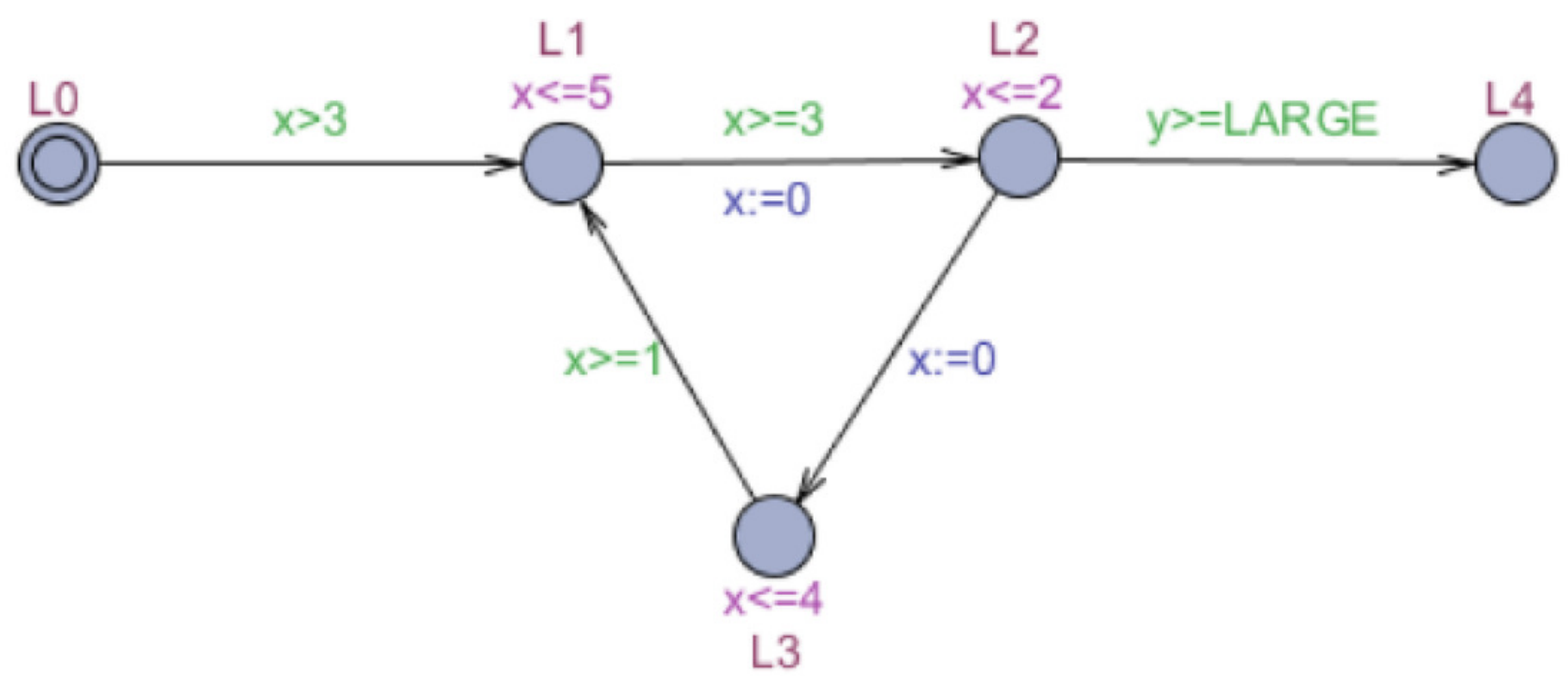


Figure 2

Accelerated automaton $M_{a}$ based on appended cycle.

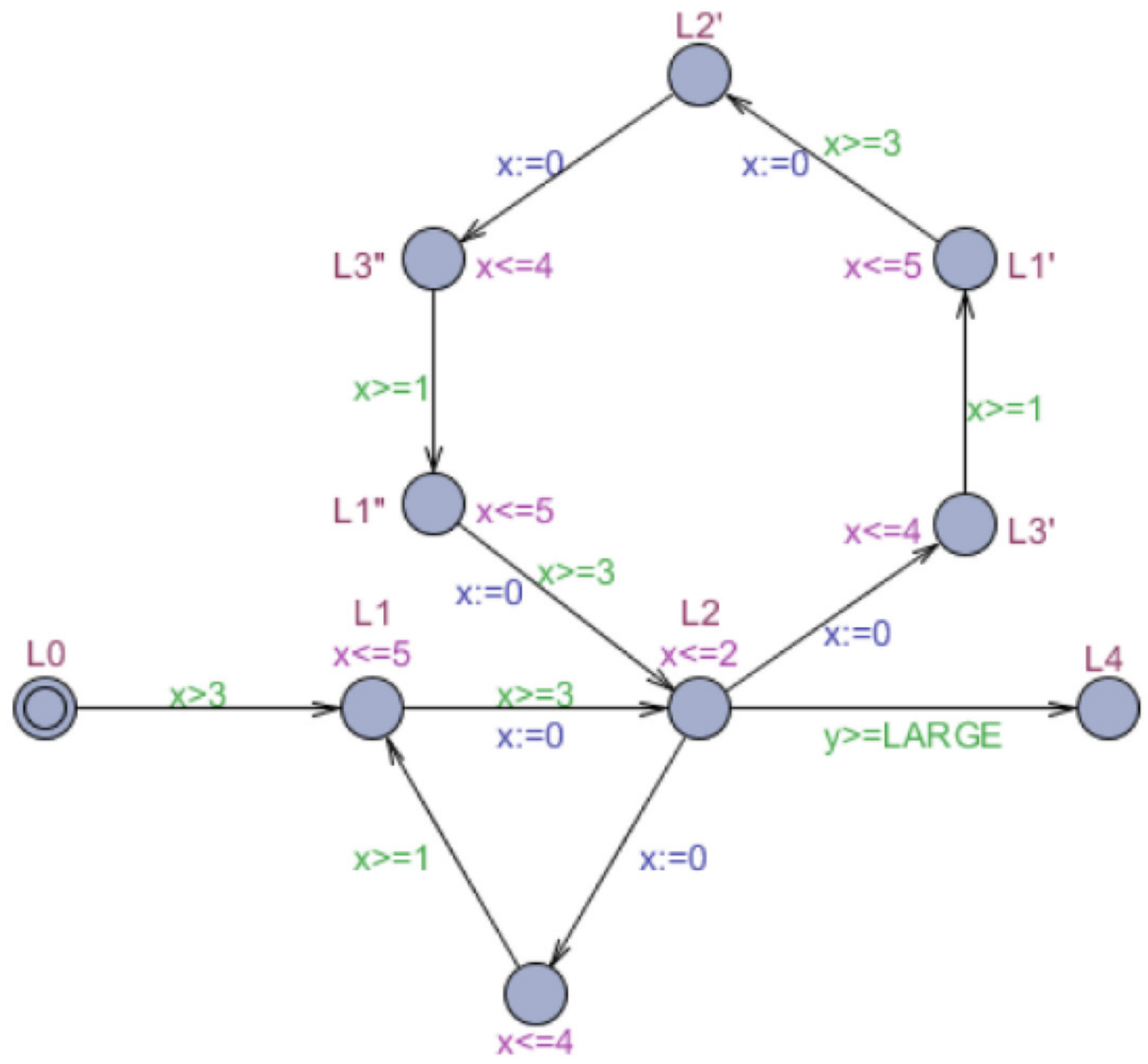

L3 
Figure 3

Accelerated automaton $M_{p}$ based on parking cycle.

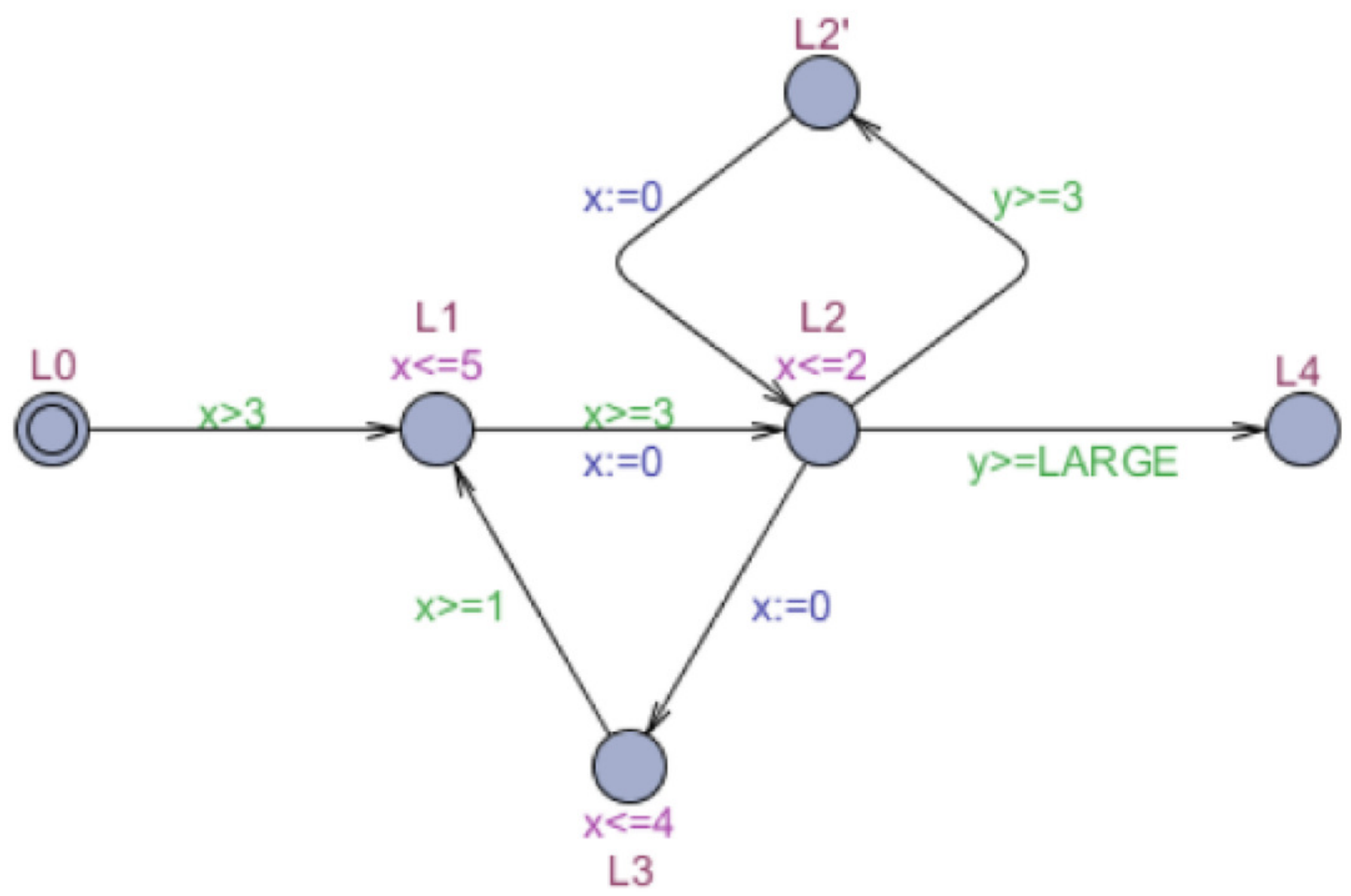


Figure 4

Timed automaton $M^{\prime}$. 


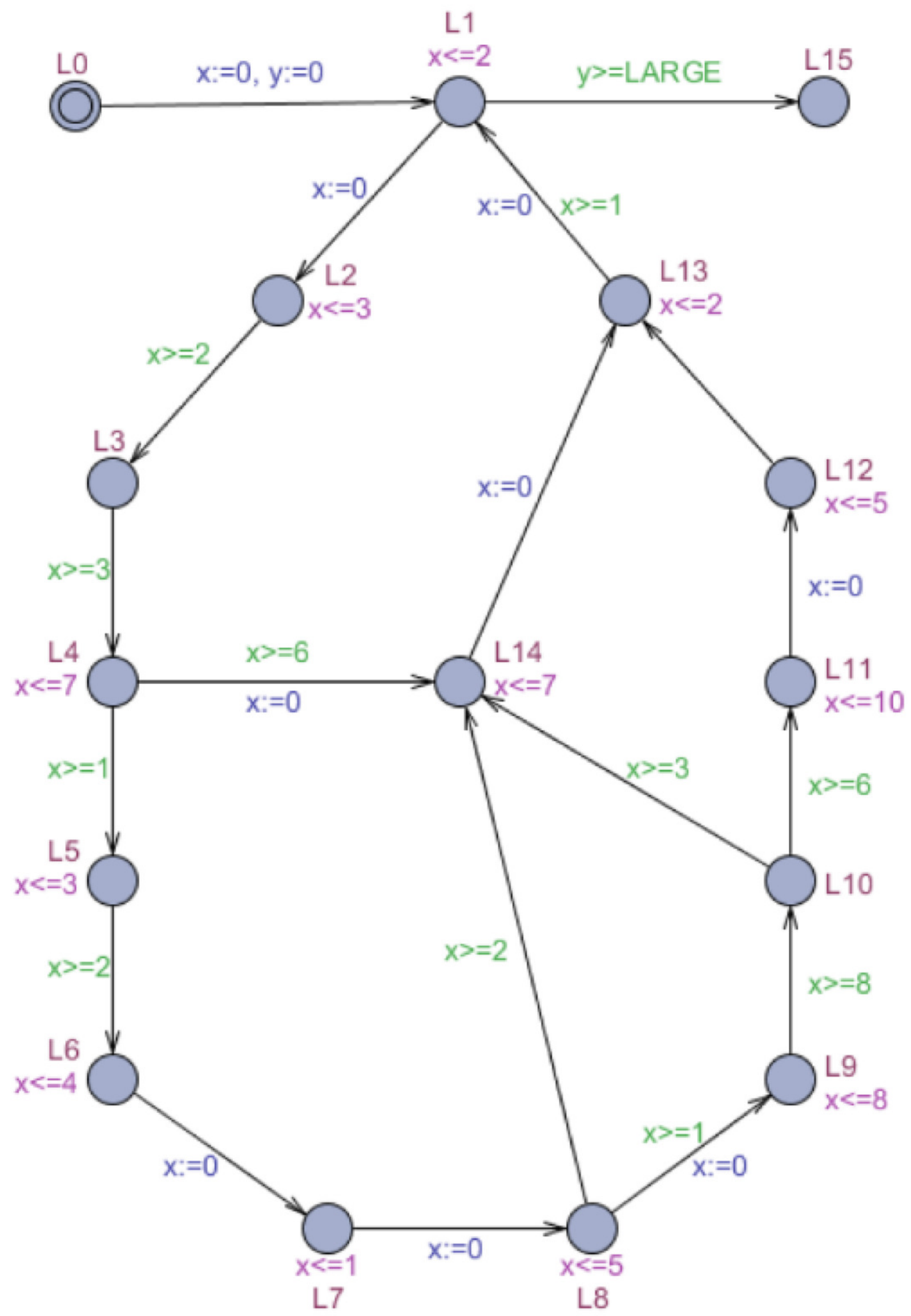


Figure 5

Automaton $M^{\prime}{ }_{0}$ : Acceleration of $M^{\prime}$ based on an overlapping cycle. 


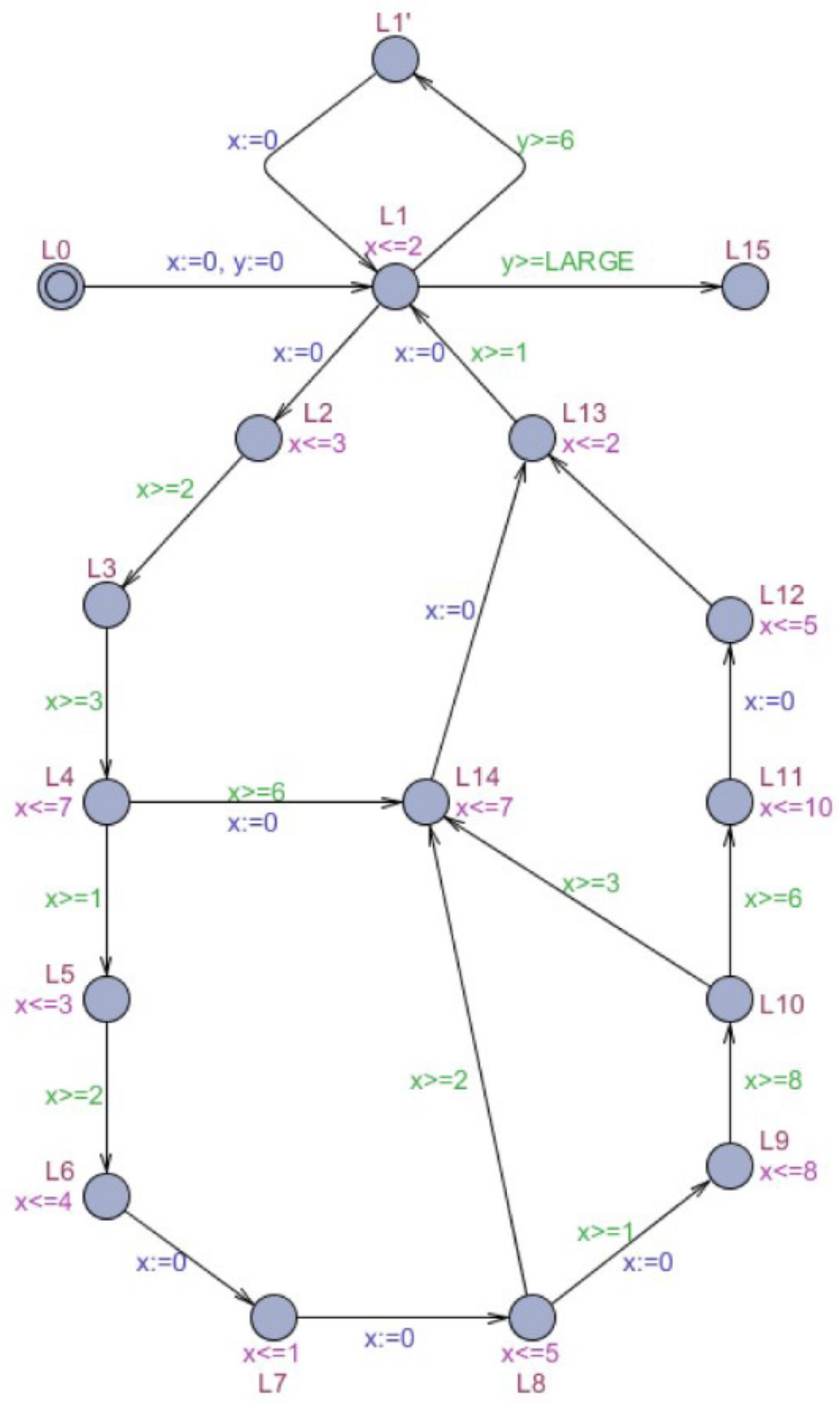


Figure 6

Technical framework of loT gateway security system.

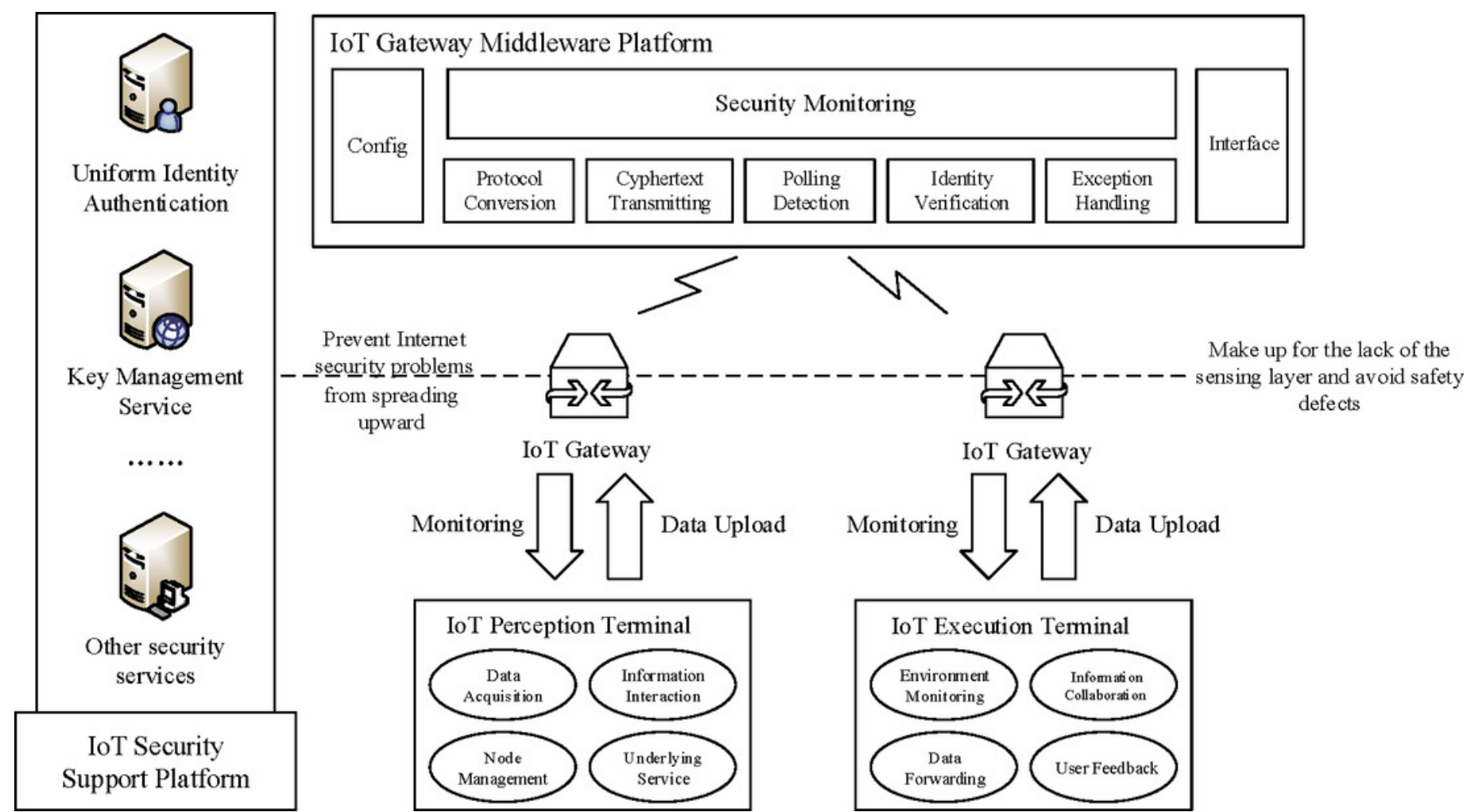


Figure 7

\section{Part of the IoT gateway security system model.}
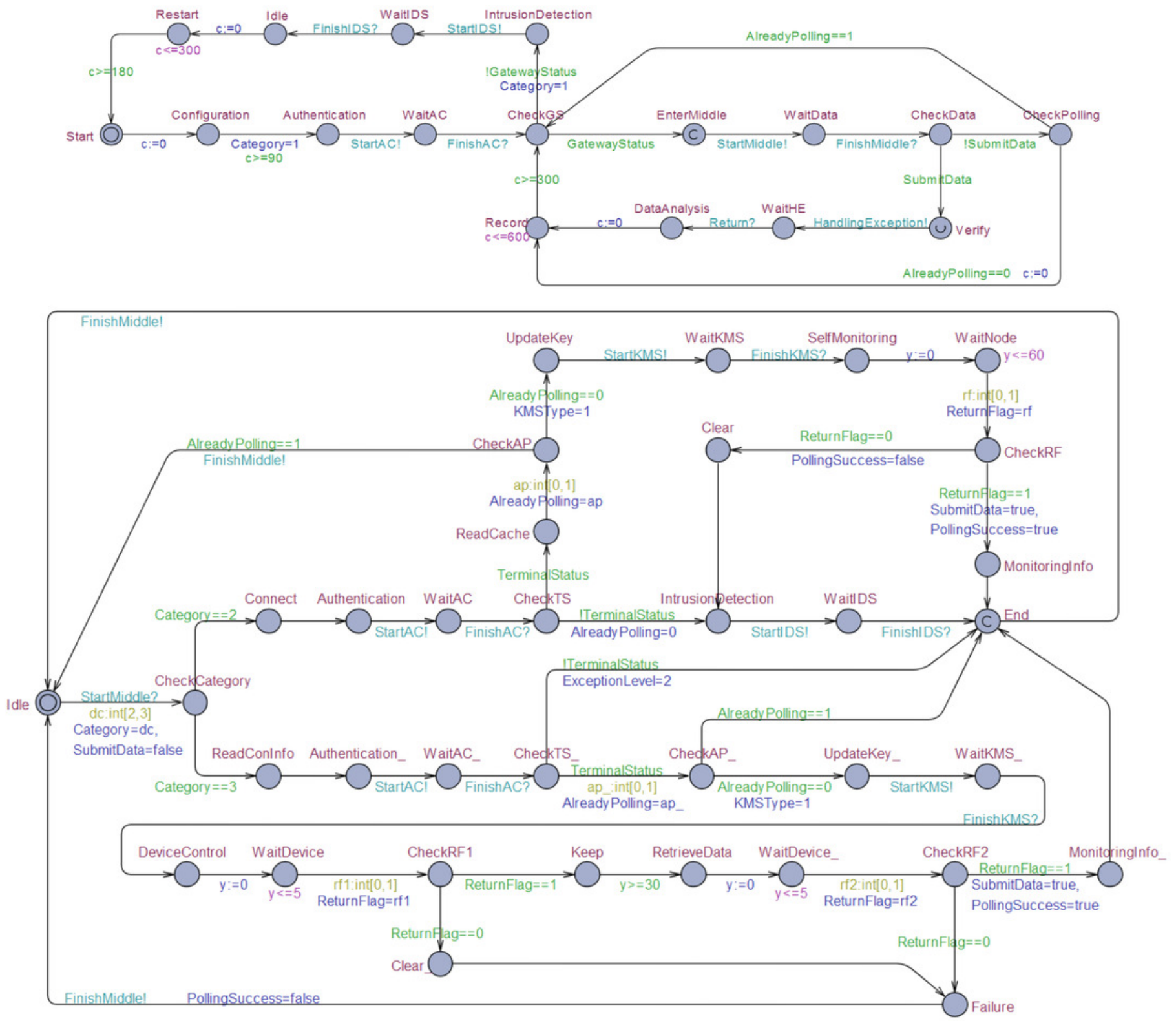


\section{Table $\mathbf{1}$ (on next page)}

Results of symbolic states from a forward symbolic exploration by timed automaton $M$. 
1 Table 1. Results of symbolic states from a forward symbolic exploration by timed automaton $2 M$.

\begin{tabular}{ccccc}
\hline State & Location & \multicolumn{3}{c}{ Zone } \\
\hline 1 & L0 & $y=0$ & $x=0$ & $y-x=0$ \\
2 & L1 & $3<y \leq 5$ & $3<x \leq 5$ & $y-x=0$ \\
3 & L2 & $3<y \leq 7$ & $0 \leq x \leq 2$ & $3<y-x \leq 5$ \\
4 & L3 & $3<y \leq 11$ & $0 \leq x \leq 4$ & $3<y-x \leq 7$ \\
5 & L1 & $4<y \leq 12$ & $1 \leq x \leq 5$ & $3<y-x \leq 7$ \\
6 & L2 & $6<y \leq 14$ & $0 \leq x \leq 2$ & $6<y-x \leq 12$ \\
7 & L3 & $6<y \leq 18$ & $0 \leq x \leq 4$ & $6<y-x \leq 14$ \\
8 & L1 & $7<y \leq 19$ & $1 \leq x \leq 5$ & $6<y-x \leq 14$ \\
9 & L2 & $9<y \leq 21$ & $0 \leq x \leq 2$ & $9<y-x \leq 19$ \\
10 & L3 & $9<y \leq 25$ & $0 \leq x \leq 4$ & $9<y-x \leq 21$ \\
11 & L1 & $10<y \leq 26$ & $1 \leq x \leq 5$ & $9<y-x \leq 21$ \\
12 & L2 & $12<y \leq 28$ & $0 \leq x \leq 2$ & $12<y-x \leq 26$ \\
\hline
\end{tabular}

3 


\section{Table 2 (on next page)}

Runtime data comparing $M^{\prime}$ and its accelerated versions $M^{\prime}{ }^{\prime}{ }^{\prime}{ }_{p}{ }_{p}$ and $M^{\prime}$. 
1 Table 2. Runtime data comparing $M^{\prime}$ and its accelerated versions $M_{a}{ }^{\prime}, M_{p}{ }^{\prime}$ and $M_{o}{ }^{\prime}$.

\begin{tabular}{cccccccc}
\hline & & \multicolumn{6}{c}{ LARGE } \\
& & $10^{4}$ & $10^{5}$ & $10^{6}$ & $10^{7}$ & $10^{8}$ & $10^{9}$ \\
\hline \multirow{2}{*}{$M^{\prime}$} & Mem (KB) & 27020 & 26892 & 27392 & 27544 & 27952 & 28572 \\
& Time (s) & 0.032 & 0.256 & 1.688 & 12.045 & 120.33 & 1191.02 \\
\multirow{2}{*}{$M_{a}^{\prime}$} & Mem (KB) & 28328 & 29220 & 30468 & 31688 & 32508 & 34104 \\
& Time (s) & 0.007 & 0.008 & 0.008 & 0.008 & 0.009 & 0.009 \\
$M_{p}^{\prime}$ & Mem (KB) & 27184 & 27384 & 27788 & 28060 & 28208 & 29276 \\
& Time (s) & 0.005 & 0.004 & 0.003 & 0.003 & 0.003 & 0.004 \\
$M_{o}^{\prime}$ & Mem (KB) & 27164 & 27036 & 27472 & 27824 & 27988 & 28788 \\
& Time (s) & 0.002 & 0.002 & 0.002 & 0.003 & 0.002 & 0.002 \\
\hline
\end{tabular}

2 


\section{Table 3(on next page)}

Runtime data comparing $M_{\text {IoT }}$ and $M_{\text {IoTo. }}$ 
1 Table 3. Runtime data comparing $M_{I o T}$ and $M_{I o T} \cdot$

\begin{tabular}{cccc}
\hline & Explored & Time(s) & Memory(KB) \\
\hline$M_{I o T}$ & 108302 & 71.151 & 29660 \\
$M_{\text {IOT }}$ & 47545 & 1.049 & 30840 \\
\hline
\end{tabular}

2 


\section{Table 4 (on next page)}

Comparing the performance of different exact acceleration techniques for large-scale IoT systems. 
1 Table 4. Comparing the performance of different exact acceleration techniques for large2 scale IoT systems.

\begin{tabular}{ccc}
\hline $\begin{array}{c}\text { System } \\
\text { States-scale }\end{array}$ & $\begin{array}{c}\text { Exact Acceleration } \\
\text { Technique }\end{array}$ & $\begin{array}{c}\text { Verification } \\
\text { Time(s) }\end{array}$ \\
\hline $10^{4}$ & Appended cycle & 277.860 \\
$10^{4}$ & Parking cycle & 0.893 \\
$10^{4}$ & Overlapping cycle & 0.015 \\
$10^{5}$ & Appended cycle & $\infty$ \\
$10^{5}$ & Parking cycle & 72.218 \\
$10^{5}$ & Overlapping cycle & 1.020 \\
$10^{6}$ & Parking cycle & 364.720 \\
$10^{6}$ & Overlapping cycle & 43.292 \\
$10^{7}$ & Parking cycle & $\infty$ \\
$10^{7}$ & Overlapping cycle & 409.132 \\
\hline
\end{tabular}

3

4 